\title{
Cognitive Conflict Is an Example of Action-Grounded Cognition
}

\author{
Sandro Rubichi (Corresponding author) \\ Department of Communication and Economics \\ University of Modena and Reggio Emilia \\ Via Allegri 8, Reggio Emilia, 42100, Italy \\ Tel: 39-522-523-224 E-mail: rubichi@unimore.it
}

\author{
Lucia Riggio
}

Department of Neuroscience, University of Parma

Via Volturno 39, Parma, 43100, Italy

Tel: 39-521-033-946 E-mail: riggio@unipr.it

Elena Gherri

Department of Psychology, City University

Northampton Square, EC1V 0HB London, UK

Tel: 44-207-040-0267 E-mail: elena.gherri.1@city.ac.uk

Roberto Nicoletti

Department of Communication, University of Bologna

Via Azzo Gardino 23, 40122-Bologna, Italy

Tel: 39-51-209-2222Ｅ-mail: roberto.nicoletti@unibo.it

Received: November 8, $2010 \quad$ Accepted: December 19, $2010 \quad$ doi:10.5539/ijps.v3n1p28

The research is funded by MIUR PRIN project 2008ZN5J5S

\begin{abstract}
The aim of the present work was to show that cognitive conflict, an issue that has been widely studied within the boundaries of the classical cognitive approach, is a clear example of higher order cognition tied to perception and action. Examples of how the cognitive conflict arising from spatial correspondence tasks is highly grounded in body attributes and in environmental/situational factors are provided. Spatial performance is strongly modulated by handedness, prior experience and by social factors. In addition, in two experiments empirical findings are reported showing that the spatial correspondence effect is a function of the location of the dynamic event even when target location is in the opposite position. These results point to the notion that spatial performance is refractory from the intervention of higher order cognition.
\end{abstract}

Keywords: Spatial performance, Embodied cognition, Grounded cognition, Simon effect, Spatial compatibility, Cognitive conflict

\section{Introduction}

Recently, there has been growing interest to explore the relations between higher order cognition and lower order operations of the perceptual and motor systems. Even if there are many theories within this approach (see e.g. Anderson, 2008), the basic idea is that cognition is grounded in action. In the present paper it is our intention to show that cognitive conflict, an issue that has been widely studied within the boundaries of the classical cognitive approach, is a clear example of higher order cognition tied to perceiving and acting. 
Cognitive conflict researchers study the effects of habitual responses on performance. Cognitive conflict is indexed by the automatic activation of a response that might be correct or incorrect. When the automatically activated response is correct, performance is faster and more accurate respect to when it should be inhibited in favor of the alternative, correct response. Well-known paradigms used to study the effects of habitual responses on performance are the spatial correspondence effects, namely the spatial stimulus-response compatibility (SRC) effect (Fitts \& Seeger, 1953) and the Simon effect (Simon \& Rudell, 1967; for a review see Proctor \& Vu, 2006). The classic situation in which both effects are evident is a choice-reaction task where the stimulus appears in a right or left location and the response is produced by one of the hands, placed on right and left keys. In the spatial SRC task, the task-relevant stimulus attribute is its location. In the compatible condition participants are instructed to respond with the key that is located on the same side as the stimulus, whereas in the incompatible condition participants are instructed to respond with the key that is on the opposite side of the stimulus. In the Simon task, the task-relevant stimulus attribute is non-spatial (e.g., form or color), and participants respond with right and left keypresses to stimuli that appear in right and left locations. Thus, there are trials in which stimulus and response locations correspond and trials in which they do not.

In both spatial tasks, performance is faster and more accurate when there is spatial correspondence between stimulus and response than when there is not. Thus, stimulus location primes automatically the spatial corresponding response even if this stimulus attribute is irrelevant for the selection of the correct response. Explanations of the spatial correspondence effects are provided within the boundaries of the classical cognitive approach. Even interpretations which emphasize the unitary of perception and action use concepts as stimulus-response "representations" or "codes" to model human performance in cognitive conflict situations. Widely accepted are dual-route models (e.g., De Jong, Lang \& Lauber, 1994; Kornblum, 1994), in which the influence of the spatial stimulus feature on performance is believed to be the result of the interaction between two parallel and independent processing routes connecting perception to action (e.g., De Jong, Lang \& Lauber, 1994; Kornblum, 1994). One route, defined as "conditional", is based on short-term, task-defined associations between stimulus and response. The other, defined as "unconditional", is based on long-term associations, which are supposed to be either genetically determined or overlearned as a result of extensive practice (e.g., Barber \& O'Leary, 1997; Zorzi \& Umiltà, 1995). Indeed, in real life, instances of corresponding S-R associations are more frequent than instances of non-corresponding S-R associations. When a stimulus appears, while the conditional route activates the response defined by task instructions, the unconditional route activates the response that spatially corresponds to the stimulus location. When the two responses correspond, no competition arises, rather the redundancy of the same response could generate a facilitation effect (Umiltà, Rubichi, \& Nicoletti, 1999). On the contrary, when they are different, the incorrect response needs to be aborted thus causing a slowing of response time and an increased number of errors.

The first part of the present work describes the most relevant examples of how the cognitive conflict arising from spatial correspondence tasks is highly tied to motor competencies and to environmental/situational factors. We will show how spatial performance is not invariant and depends on handedness, prior experience and on social factors. We will describe how the magnitude of the Simon effect is modulated by these factors. In the second part of the paper we report empirical findings with a Simon task with onset and offset stimuli which indicate how spatial performance is refractory from the intervention of higher order cognition.

\subsection{Motor competencies modulations: The effect of handedness}

Rubichi and Nicoletti (2006) evaluated the influence of motor competencies on the Simon effect. In particular, they studied the relation between handedness and the size of the Simon effect in each visual hemifield. They found that the Simon effect was larger in the right visual hemifield in right-handers and in the left visual hemifield in left-handers, whereas ambidextrous individuals showed a symmetric Simon effect. When participants performed the Simon task with crossed hands, the right- and left-handers group showed a reversed pattern of results. Since conflict resolution processes in spatial tasks are intrinsically related to the position where the dominant hand operates, this pattern of results clearly indicate that cognitive conflict depends on body's steady and transient features.

\subsection{Environment related modulations: Practice and sequential effect}

Cognitive conflict indexed by the Simon effect has proven to be a robust phenomenon, whose magnitude can be only partially reduced through repeated practice (e.g., Proctor \& Lu, 1999; Simon, Craft, \& Webster, 1973). However, recent findings suggest that it can be strongly modulated by the prior experience, both at a betweenand within-task level.

When we consider the between-task level, we refer to the works that assessed performance on the Simon task as 
a function of practice with a different task (from now on, practice effects). These works indicate that while practicing with a spatially compatible mapping (e.g., respond to the left stimulus with the left key and vice versa) before performing a Simon task has a minimal effect on the magnitude of the Simon effect, practicing with an incompatible spatial mapping (e.g., respond to the left stimulus with the right key and vice versa) can eliminate (Tagliabue, Zorzi, Umiltà, \& Bassignani, 2000; Iani, Rubichi, Gherri, \& Nicoletti, 2009) or even reverse the Simon effect (Proctor \& Lu, 1999). To note, a recent work showed that modulations of spatial performance also occur for motor behaviors acquired in real life (Pellicano, Iani, Rubichi, Ricciardelli, Borghi, \& Nicoletti, 2010). In this work performance of skilled drummers was compared to that of non-drummers and non-musicians in the Simon and SRC tasks, to investigate whether and to what extent spatial performance can be modified by motor behaviors acquired in real life. Drummers were chosen because, compared to other musicians and to the general population, their efficient performance mainly depends on the processing of spatial information. The spatial SRC effect was less pronounced in drummers. The advantage was present even when feet were used as responding effectors, suggesting that the influence of motor behaviors is generalized and does not depend on the use of specific muscles patterns.

At a within-task level, the Simon effect reverses when location-relevant trials with an incompatible mapping and location-irrelevant trials are intermixed in the same task (Marble \& Proctor, 2000; Proctor, Marble, \& Vu, 2000; Notebaert, Gevers, Verbruggen, \& Liefooghe, 2006), or when noncorresponding trials are $80 \%$ of the total trials, while it increases when corresponding trials predominate (Toth, Levine, Stuss, Oh, Winocur, \& Meiran, 1995). With an equal number of corresponding and non-corresponding trials, it has been shown to reduce (e.g. Praamstra, Kleine \& Schultz, 1999; Ridderinkhof, 2002), disappear (e.g. Mordkoff, 1998; Stürmer, Leuthold, Soetens, Schröter \& Sommer, 2002) or even reverse (e.g. Di Pellegrino, Ciaramelli, \& Làdavas, 2007; Wendt, Kluwe, \& Peters, 2006) after a non-corresponding trial, whereas a regular effect is evident after a corresponding trial. These latter phenomena are referred to as sequential effects (see also Iani, Rubichi et al. 2009) and indicate that the Simon effect is not an invariant phenomenon. Stimulus-response translations vary each trial depending on the nature of the trial immediately before.

Taken together, these results suggest that the influence of irrelevant spatial information on performance is not as unavoidable as once thought, but rather depends on the preceding experience and on the specific task context.

\subsection{Situation related modulations: post-hypnotic suggestions and the interactive Simon effect}

A recent work was designed to assess whether the interference exerted by task-irrelevant spatial information might be modulated by means of a specific posthypnotic suggestion (Iani, Ricci, Baroni, \& Rubichi, 2009). To this purpose high and low-susceptible participants were tested using a Simon task with central arrows after the administration of a suggestion aimed at preventing the processing of the irrelevant spatial information conveyed by the arrow. The Simon effect was significantly reduced following the posthypnotic suggestion in high-susceptible participants. These results show that a specific posthypnotic suggestion can alter information processing in high-susceptible individuals and reduce the interfering effect exerted by arrow stimuli.

It is also well-established that spatial performance depends on whether the agent is performing alone or with another agent. In a study by Sebanz, Knoblich and Prinz (2003), participants were shown photographs of a centrally presented right hand pointing to the right, to the left, or straight, with the instruction to press one of two lateralized keys according to the color of a ring appearing on the index finger. They were required to perform the task either alone or paired with another participant (interactive Simon task). The advantage for corresponding trials (i.e., Simon effect) was absent when each participant performed the task alone with the instruction to respond to only one color, but was present when participants performed the task alongside another participant, each responding to only one color. To note, it has been showed that also the practice effects described in the preceding section are modulated by the presence of another co-actor (Milanese, Iani, \& Rubichi, 2010). On the whole, these results point to the notion that situational factors modulate the cognitive conflict occurring in spatial performance.

The phenomena described so far clearly indicate that cognitive conflict in spatial performance is determined by bodily states, environment and situations in which spatial performance takes place. In the present study we hypothesize that if what determines cognitive conflict are processes related to bodily, environmental and situational states, spatial performance should be substantially refractory from the intervention of higher cognitive processes.

To this end, we adopted the paradigm introduced by Wühr \& Kunde (2006) which studied spatial performance in a Simon task with onset and offset stimuli. To clarify, the onset condition is when participants respond to the appearance of a lateralized stimulus, while the offset condition is when participants respond to the disappearance 
of a lateralized stimulus. In this latter condition, the trial starts with two lateralized stimuli on the screen. The authors found the Simon effect with both stimulus onset and stimulus offset situations. These results indicate that the Simon effect occurs in relation to the dynamic event which takes place in the environment, being it the appearance or the disappearance of a stimulus. In Experiment 1 participants performance was compared in onset and offset Simon tasks. To dissociate the target stimulus from the dynamic event, participants of Experiment 2 were required to respond to the stimulus that remained on the screen after the offset event. If, contrary to our hypothesis, higher cognitive processes mediating task instructions do overcome the relevance of the dynamic event, then the Simon effect should occur in relation to the location of the target stimulus that is made relevant by instructions, irrespective of the stimulus which determines the dynamic event.

\section{Experiment 1}

In this experiment the target stimulus was an onset or an offset of a geometrical figure. Participants had to respond on the basis of the shape of the figure that either appeared (onset trials) or disappeared (offset trials). As in Wühr and Kunde (2006), the dynamic event (i.e., stimulus onset or stimulus offset) always coincided with the target stimulus, that is, the stimulus relevant for response selection. This experiment allowed us to evaluate the Simon effect for onset and offset stimuli.

\subsection{Material and Methods}

\subsubsection{Participants}

24 right-handed participants, 12 males and 12 females, between 20-31 years of age, volunteered to take part in the experiment. They had normal or corrected-to-normal vision and were naive as to the purpose of the experiment. Hand preference was assessed according to the Edinburgh Handedness Inventory (Oldfield, 1971).

\subsubsection{Apparatus and display}

The experiment took place in a sound-attenuated room, dimly illuminated by a halogen lamp directed towards the ceiling. Stimuli were displayed on a VGA monitor with P22 phosphor driven by an IBM-compatible PC, running MEL software (Schneider, 1988) that generated the stimuli, controlled timing operations, and recorded the responses. The experimental monitor was mounted in a wooden frame and was covered by a gray cardboard, except for an $18 \times 13 \mathrm{~cm}$ window in which the stimuli were displayed. Participants sat in front of the monitor with the head positioned in an adjustable head-and-chinrest, so that the distance between the eyes and the screen was approximately $57 \mathrm{~cm}$. Eye height was adjusted to the level of fixation.

Stimuli were two white geometrical figures, a rectangle and a diamond having the same area (1.5 X 1.5$)$, displayed on a dark-black background. These figures were displayed $5^{\circ}$ (center to center) to the right or to the left of the fixation cross $(0.3 \times 0.3)$.

Response keys were the two shift keys of a keyboard, aligned with the center of the screen; in this way one key was located to the right of the body midline and was pressed by the right index finger, whereas the other key was located to the left and was pressed by the left index finger. During the experiment the index fingers were held on the response keys.

Since dynamic events elicit eye movements to foveate the target, eye movements were monitored in order to delete trials in which eye movements were executed. Eye position was monitored using $10-\mathrm{mm}$ electrodes located at the outer canthus of each eye. The electrodes were referred to an electrode attached to the right ear-lobe. Electro-oculographic (EOG) signals were digitized (sampling rate of $100 \mathrm{~Hz}$ ) after filtering $(0.5-2.0 \mathrm{~Hz}$, $12 \mathrm{~dB}$ octave) and high gain amplification $\left(10^{4}\right)$. An IBM-compatible PC controlled data acquisition. Before the experiment, participants had to execute some calibration trials, which consisted in the $2^{\circ}$-pursuit-movements, in both horizontal directions, of a moving spot stimulus on the computer screen. This procedure allowed us to determine, for each participant, the baseline of 2-movements. The output of the EOG, after on-line smoothing, which used a three-points moving average, was displayed on the monitor of an IBM-compatible PC, were two horizontal lines indicated the extent of 2-movement to the right and to the left of the fixation point. An EOG signal that reached or overcame the baselines indexed eye movements equal or greater than 2 . The corresponding trial was rejected and rerun at the end of the session. The experimenter, the monitoring eye movement apparatus and the computer that controlled the experiment were located in a room next to the experimental room.

\subsubsection{Procedure}

Each participant performed both the onset and the offset conditions. Order of the conditions was balanced across participants; half of them began with the onset followed by the offset condition. The reverse was true for the other participants. 
In the onset condition, which is a standard Simon task, each trial began with the presentation of the fixation cross, shown at the geometrical center of the screen along with an auditory warning signal. After $1000 \mathrm{~ms}$ the target (a rectangle or a diamond) was presented to the right or to the left of fixation and remained on the screen until response was given, but anyway no longer than $1000 \mathrm{~ms}$.

In the offset condition, at the beginning of each trial along with the fixation and the auditory warning signal, both geometrical shapes (the diamond and the rectangle) were presented (one to the right and the other to the left of the fixation cross). After $1000 \mathrm{~ms}$, the target-stimulus (i.e., one of the two shapes) disappeared.

Participants were instructed to respond to the identity of the target event which was the appearance and the disappearance of one of the two stimuli in the onset and in the offset conditions, respectively. They were also instructed to maintain gaze at fixation. Half of the participants pressed the right key for the onset or the offset of the diamond and the left key for the onset or the offset of the rectangle, while the other half of the participants had the opposite pairings.

After the response a feedback about reaction time (RT) and accuracy was provided at the center of the screen. Trials for which RT was less than $150 \mathrm{~ms}$ (anticipations) or more than $1000 \mathrm{~ms}$ (missing responses) were discarded and later rerun, as well as trials in which the wrong key was pressed.

The experiment consisted of 160 correct trials for the onset condition and 160 for the offset condition, each subdivided into four blocks of 40 trials. Target appearance or disappearance occurred according to a quasi-random sequence, with the constraints that both the rectangle and the diamond appeared or disappeared, half the time to the right, and half to the left of the fixation cross. Each condition was preceded by one block of practice trials. Two variables were considered according to the type of target (onset vs. offset) and S-R correspondence (corresponding vs. noncorresponding). 80 correct trials were provided for each combination of the experimental variables.

\subsection{Results and discussion}

Overall errors (anticipations, missing responses, key errors and eye-movements) were less than $6 \%$. Key error percentages were arcsine-transformed and entered into an analysis of variance (ANOVA) with type of Target (onset vs. offset), and S-R Correspondence (corresponding vs. noncorresponding), as within-participants variables. The main effect of Correspondence, $\mathrm{F}(1,23)=6.54, \mathrm{MSE}=0.007, \mathrm{p}<0.02$, was significant with more errors in noncorresponding than in corresponding trials ( $4.8 \%$ vs. $3.1 \%)$.

The same ANOVA, computed on mean RTs, showed significant main effects of type of Target, $F(1,22)=45.5$, $\mathrm{MSE}=9217.89, \mathrm{p}<0.001$, and Correspondence, $\mathrm{F}(1,22)=5.6, \mathrm{MSE}=2239.42, \mathrm{p}<0.03$. RTs were faster in the offset condition $(432 \mathrm{~ms})$ than in the onset condition $(491 \mathrm{~ms})$. The Simon effect was of $10 \mathrm{~ms}$ for the onset condition (corresponding trials: $486 \mathrm{~ms}$; noncorresponding trials: $496 \mathrm{~ms}$ ) and of $11 \mathrm{~ms}$ for the offset condition (corresponding trials: $427 \mathrm{~ms}$; noncorresponding trials: $438 \mathrm{~ms}$ )

As in Wühr and Kunde (2006), the present experiment showed the Simon effect in the offset condition. The Simon effect in the offset condition clearly showed that the presence of an object-is not mandatory for the phenomenon itself, with the consequence that target location can be empty at the moment of response selection.

\section{Experiment 2}

In the present experiment, we tested whether the Simon effect occurs in relation to the location of the target stimulus that is made relevant by instructions, irrespective of the stimulus which determines the dynamic event. Indeed, while in the previous experiment the target was the figure that disappeared, in this experiment the target was the remaining stimulus. As a consequence, the dynamic event and the target relevant for the response are located on opposite sides of the fixation cross.

If what matters for the Simon effect is the location of the dynamic event, the same pattern of results obtained in the offset condition of the previous experiment was expected, whereas if what matters is the target location defined by instructions, the opposite pattern of results should be obtained.

\subsection{Material and Methods}

\subsubsection{Participants}

18 right-handed participants, 5 males and 13 females, 20-28 years of age, who had not participated in the previous experiment, volunteered to take part in this experiment. All had normal or corrected-to-normal vision and were naive as to the purpose of the experiment.

\subsubsection{Apparatus, display and procedure}


They were the same as the offset condition of Experiment 1, except that participants were instructed that the target stimulus was the figure which remained on the screen.

\subsection{Results and discussion}

Overall errors (anticipation, missing response, key error and eye-movement) were less than 6\%. Key error percentages were arcsine-transformed and entered into an ANOVA with Correspondence as within-participants variable. Errors were quite similar in the corresponding and noncorresponding trials ( $4.5 \%$ vs. $4.3 \%)$, and they did not significantly differ.

Also correct RTs were analyzed with the same ANOVA, showing the main effect of Correspondence, $\mathrm{F}(1,17)=$ 31.9, MSE $=1405.89, \mathrm{p}<0.001$. There was a reversed Simon effect of $32 \mathrm{~ms}$ (corresponding trials: $566 \mathrm{~ms}$; noncorresponding trials: $534 \mathrm{~ms}$ )

In the offset Simon task adopted by the present experiment the target was the stimulus that remained on the screen after the offset of the other stimulus in the opposite location. Results showed that the Simon effect was a function of the spatial correspondence between the locations of stimulus that went off and the response. More precisely, RTs were faster when response location corresponded with offset stimulus location than when it corresponded with target location. This result closely resembles that found in the offset condition of Experiment 1 when participants were asked to respond to the identity of the offset stimulus. Thus, what matters for the Simon effect is the location of the dynamic event, even when the target stimulus specified by the instructions is the remaining stimulus in the opposite location.

\section{Conclusions}

The present study was aimed at showing that cognitive conflict is an example of higher order cognition grounded in perception and action. In the first part we provided examples that spatial performance is determined by motor competencies and environmental/situational states. We reported studies with modulations of spatial performance with respect to handedness, prior experience and social factors.

The second part of the paper provided empirical support to the notion that spatial performance is refractory from the intervention of higher order cognition. In Experiment 1 we replicated the results of Wühr and Kunde (2006), that is, the Simon effect was present for both onset and offset conditions. The onset condition was a typical Simon task in which the location of the dynamic event coincides with the target location. In Experiment 2, the target location and the location of the dynamic event were in opposite positions. We reasoned that if higher order cognition determines spatial performance, the Simon effect should occur in relation to the target stimulus. On the contrary, it should occur always in relation to the dynamic event. Both in Experiments 1 and 2 the Simon effect was a function of the offset stimulus. On the whole these results showed that what really matters is the location of the dynamic event and thus support the idea that no modulations due to higher mental processes take place. The interest to show that cognitive conflict is action-grounded arises because, besides perceptual and motor processes, decision processes are involved. The observations that we provided in the present work point to the idea that decision processes are inherently tied to, that is grounded in, perceiving and acting and their activity is strongly modulated by bodily and environmental/situational states.

\section{References}

Anderson, M.L. (2008). On the ground of x-grounded cognition. In P. Calvo, \& T. Gomila (Eds.), The Elsevier Handbook of Cognitive Science: An Embodied Approach. Amsterdam: Elsevier pp. 423-35.

Barber, P., \& O'Leary, M. (1997). The relevance of salience: Towards an activation account of irrelevant stimulus-response compatibility effects. In. B. Hommel, \& W. Prinz (Eds.), Theoretical Issues in Stimulus-Response Compatibility. Amsterdam: North-Holland pp. 135-172.

De Jong, R., Liang, C.-C., \& Lauber, E. (1994). Conditional and unconditional automaticity: A dual-process model of effects of spatial stimulus-response correspondence. Journal of Experimental Psychology: Human Perception and Performance, 20, 731-750.

Di Pellegrino, G., Ciaramelli, E., \& Làdavas, E. (2007). The regulation of cognitive control following rostral anterior cingulate cortex lesions in humans. Journal of Cognitive Neuroscience, 19, 275-286.

Fitts, P.M., \& Seeger, C.M. (1953). S-R compatibility: Spatial characteristics of stimulus and response codes. Journal of Experimental Psychology, 46, 199-210.

Kornblum, S. (1994). The way irrelevant dimensions are processed depends on what they overlap with: The case of Stroop- and Simon-like stimuli. Psychological Research, 56, 130-135. 
Iani, C. Ricci, F. Baroni, G., \& Rubichi, S. (2009). Attention control and susceptibility to hypnosis. Consciousness and Cognition, 18, 856-863.

Iani, C., Rubichi, R., Gherri, E., Nicoletti, R. (2009). Co-occurrence of sequential and practice effects in the Simon task: Evidence for two independent mechanisms affecting response selection. Memory \& Cognition, 37, 358-367.

Marble, J.G., \& Proctor, R.W. (2000). Mixing location-relevant and location-irrelevant choice-reaction tasks: Influences of location mapping on the Simon effect. Journal of Experimental Psychology: Human Perception \& Performance, 26, 1515-1533.

Milanese, N., Iani, C., \& Rubichi, S. (2010). Shared learning shapes human performance: Evidence from transfer effects in task sharing. Cognition, 116, 15-22.

Mordkoff, T. (1998). The gating of irrelevant information in selective-attention tasks. Abstracts of the Psychonomic Society, 3, 193.

Notebaert, W., Gevers, W., Verbruggen, F., \& Liefooghe, B. (2006). Top-down and bottom-up sequential modulations of congruency effects. Psychonomic Bulletin \& Review, 13, 112-117.

Oldfield, R.C. (1971). The assessment and analysis of handedness: the Edinburgh Inventory. Neuropsychologia, 9, 97-113.

Pellicano, A., Iani, C., Rubichi, S., Ricciardelli, P., Borghi, A.M., \& Nicoletti, R. (2010). Real life motor training modifies spatial performance: The advantage of being drummers. American Journal of Psychology, 123, 169-179.

Praamstra, P., Kleine, B.U., \& Schnitzler, A. (1999). Magnetic stimulation of the dorsal premotor cortex modulates the Simon effect. NeuroReport, 10, 3671-3674.

Proctor, R. W., \& Lu, C.-H. (1999). Processing irrelevant information: Practice and transfer effects in choice-reaction tasks. Memory \& Cognition, 27, 63-77.

Proctor, R.W., Marble, J.G., \& Vu, K.-P.L. (2000). Mixing incompatibly mapping location-relevant trials with location-irrelevant trials: Effects of stimulus mode in the reverse Simon effect. Psychological Research, 64, 11-24.

Proctor, R.W., \& Vu, K-P. L. (2006). Stimulus-Response Compatibility Principle: Data, theory, and Application. Boca Raton, FL: Taylor \& Francis.

Ridderinkhof, K.R. (2002). Activation and suppression in conflict tasks: Empirical clarifications through distributional analysis. In W. Prinz and B. Hommel (Eds.), Attention and Performance: Vol. XIX. Common Mechanisms in Perception and Action. Oxford, UK: Oxford University Press pp. 494-519.

Rubichi, S., \& Nicoletti, R. (2006) The Simon effect and handedness: Evidence for a dominant-hand attentional bias in spatial coding. Perception \& Psychophysics, 68, 1059-1069.

Sebanz, N., Knoblich, G., \& Prinz, W. (2003). Representing other's actions: Just like one's own? Cognition, 88, $11-21$.

Simon, J.R., Craft, J.L., \& Webster, J.B. (1973). Reactions toward the stimulus source: Analysis of correct responses and errors over a five-day period. Journal of Experimental Psychology, 101, 175-178.

Simon, J.R., \& Rudell, A.P. (1967). Auditory S-R compatibility: The effect of an irrelevant cue on information processing. Journal of Applied Psychology, 51, 300-104.

Stürmer, B., Leuthold, H., Soetens, E., Schröter, H., \& Sommer, W. (2002). Control over location-based priming in the Simon task: Behavioral and electrophysiological evidence. Journal of Experimental Psychology: Human Perception and Performance, 28, 1345-1363.

Tagliabue, M., Zorzi, M., Umiltà, C., \& Bassignani, C. (2000). The role of LTM links and STM links in the Simon effect. Journal of Experimental Psychology: Human Perception and Performance, 26, 648-670.

Toth, J.P., Levine, B., Stuss, D.T., Oh, A., Winocur, G., \& Meiran, N. (1995). Dissociation of processes underlying spatial S-R compatibility: Evidence for the independent influence of what and where. Consciousness \& Cognition, 4, 483-501.

Umiltà, C.A., Rubichi, S., \& Nicoletti, R. (1999). Facilitation and interference components in the Simon effect. Archives Italiennes de Biologie, 137, 139-149. 
Wühr, P., \& Kunde, W. (2006). Spatial correspondence between onsets and offsets of stimuli and responses. European Journal of Cognitive Psychology, 18, 359-377.

Zorzi, M., \& Umiltà, C. (1995). A computational model of the Simon effect. Psychological Research, 58, 193-205. 